\title{
Establishing a Novel In Vivo Platform to Interrogate the Effects of Pharmacologic and Molecularly Targeted Modulation of CDK5 in the Invasive and Metastatic Potential of Medulloblastoma in Live Experimental Animals
}

\author{
A. Petrosiute, J. Myers, J. Nthale, K. Davis, A.Y. Huang \\ Division of Pediatric Hematology/Oncology, Department of Pediatrics, Case Western Reserve University \\ School of Medicine, Wolstein 6401, 2103 Cornell Road, Cleveland, OH 44106-7288
}

Medulloblastoma (MB) is the most common malignant brain tumor of childhood. Originating from embryonal neuroepithelial cells, MB accounts for approximately $20 \%$ of all primary pediatric CNS tumors. MB often exhibits an aggressive growth pattern and is one of only a few tumor types with a metastatic potential. It can attain several centimeters in size resulting in mass-occupying lesions most often located in the posterior fossa. Due to invasion into regional subarachnoid and ventricular spaces, widespread seeding of the subarachnoid space may occur. Unfortunately, effective therapy for metastatic MB continues to be a great clinical challenge; therefore, insights into the mechanisms by which MB attains its invasive properties may spark future development of rational new therapeutic strategies.

Recently, Cdk5, a member of the cyclin-dependent kinase family of proteins, has been implicated in conferring migratory capacity of neurons in developing fetal brain. The involvement of Cdk5 in the migratory and metastatic behavior of MB remains uninvestigated, and is a subject of our current study. We hypothesize that $\mathrm{Cdk} 5$ plays a functional role in $\mathrm{MB}$ through modulation of the Akt signaling pathway and activation of ezrin. We further hypothesize that disruption of Cdk5 functional activities may diminish the invasive and metastatic characteristics of $\mathrm{MB}$ in vivo and in vitro. Utilizing intravital 2photon laser scanning microscopy technology available in our laboratory, we aim to directly observe MB invasion and micro-metastasis in real time in vivo, and to observe the direct effect of pharmacologic Cdk5 inactivation on tumor behavior in live mouse through serial imaging of the brain containing the developing CNS tumor.

Utilizing murine MB cell lines derived from C57B1/6 Patched $^{+/} p 53^{-/-}$mice, we show that all 5 available MB cell lines (MM1-MM5) expressed Cdk5 message and protein. However, p35 and p39 (signaling partner proteins for Cdk5 in neurons) were not consistently detected. Cyclin I was expressed three cell lines, while expressions of Akt and Ezrin were variable. We tested the ability of Cdk5 inhibitor, roscovitin, to affect local invasive properties of Cdk5-expressing MB cell lines in vitro. We are currently interrogating the effect of $\mathrm{Cdk} 5$ on $\mathrm{MB}$ formation and local invasion in vivo through direct visualization by intravital two-photon microscopy (Fig. 1A and B). To accomplish this, we have created an in vivo imaging platform to investigate how pharmacologic or molecularly targeted manipulations to modulate Cdk5 activity may affect MB biology in live experimental hosts.

Finally, by establishing a critical role for Cdk5 in regulating the invasive properties of MB, our studies will provide foundations for new rationales to pursuing Cdk5 as a therapeutic target in the treatment of this most common childhood CNS cancer. 


\section{Reference:}

[1] This work is supported by grants from the St. Baldrick's Foundation, Hyundai Motors of America "Hope-on-Wheels" Research Award, the Gabrielle's Angel Foundation, the Dana Foundation, Cancer Research Institute, Rainbow Fellow Research Award Program, and the Bert Wolstein Scholar Award. A.P is a recipient of the St. Baldrick's Post-doctoral Fellowship Award. A.Y.H. is a recipient of the St. Baldrick's Research Scholar Award.
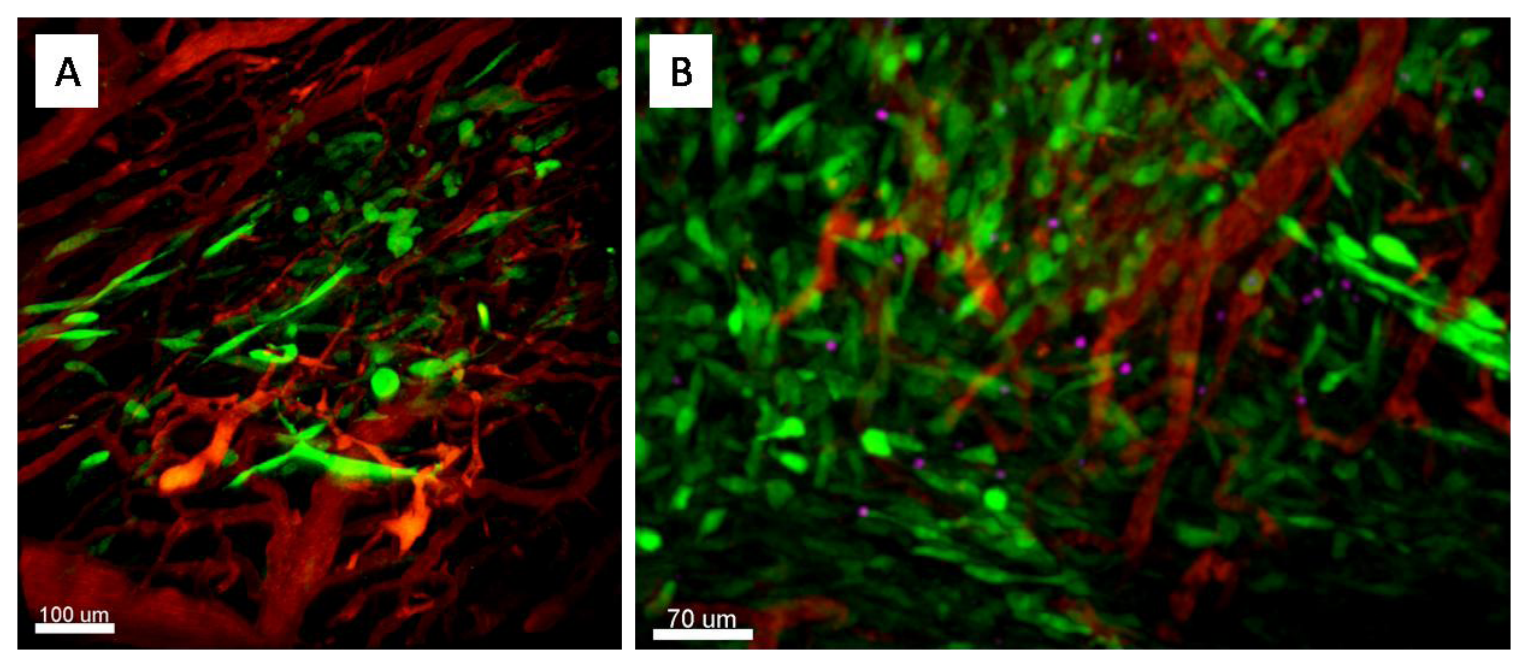

FIG. 1. MM1-GFP-OVA cells injected intra-cranially into C57BL/6 mice. A) Visualizing the relationship between MM1-GFP-OVA cells (green) the vasculature (TRITC-dextran, red) in the brain. B) MM1-GFP-OVA with SNARF labeled T-cells (magenta) and vessels. 